\title{
Development of an Algebraic Turbulence Model for Analysis of Propulsion Flows
}

N.J. Georgiadis

NASA Lewis Research Center

Cleveland, Ohio

and

J.E. Drummond and B.P. Leonard

The University of Akron

Akron, Ohio

Prepared for the

28th Joint Propulsion Conference and Exhibit

cosponsored by the AIAA, SAE, ASME, and ASEE

Nashville, Tennessee, July 6-8, 1992

\section{N/SN


The objective of the current investigation was to develop a simple turbulence model that will be applicable to propulsion flows having both bounded and unbounded regions by linking two existing algebraic turbulence models. The first is the Modified Mixing Length model, which is optimized for wall-bounded flows and has not previously been incorporated into the PARC code. The second is the Thomas model, the existing algebraic turbulence model in PARC, which has been used to calculate both bounded and unbounded turbulent flows, but was optimized for the latter. The following sections discuss both models, the method employed to link them into one model, and the validation of the resulting combination.

\section{Modified Mixing Length Model for Wall-Bounded Flows}

The Modified Mixing Length (MML) model is an algebraic turbulence model originally developed to analyze airflow over iced airfoils ${ }^{3}$ with the ARC external flow Navier-Stokes code. ${ }^{4}$ Potapczuk compared results obtained with this MML turbulence model and the Baldwin-Lomax turbulence model and found that the MML solutions more closely matched experimental data than did the BaldwinLomax solutions in separated flow situations.

The MML model calculates turbulent viscosity for wall-bounded flows through Prandtl's mixing length hypothesis

$$
\mu_{t}=\rho \ell^{2}|\omega|
$$

The mixing length $\ell$ is defined as a function of distance from the wall

$$
\begin{gathered}
\ell=K \frac{C_{1}}{C_{2}} y^{*}\left(1-\left(1-\frac{y^{+}}{C_{1}}\right)^{C_{2}}\right)\left(1-e^{\frac{-y^{+}}{A^{+}}}\right), \mathrm{y}^{+}<C_{1} \\
\ell=\ell_{\mathrm{CAP}}=K \frac{C_{1}}{C_{2}} y^{*}, \quad \mathrm{y}^{+}>C_{1}
\end{gathered}
$$

where

$$
\mathrm{y}^{*}=\left.\frac{\mu}{\sqrt{\rho|\tau|}}\right|_{w}
$$

and

$$
y^{+}=\frac{y}{y^{*}}
$$

The von Karman constant, $K=0.41$, and van Driest constant, $A^{+}=26$, are the same as in the Baldwin-Lomax turbulence model, 5 and $C_{1}$ and $C_{2}$ are empirical constants.

The expressions for the length scale in a turbulent boundary layer given in Eqs. (2) and (3) were retained for the MML model in PARC. The terms $C_{1}$ and $C_{2}$, however, were not restricted to being constants as in the original formulation; instead, their ratio was allowed to vary as a function of a local flow parameter, such as wall shear stress. An expression relating $C_{1}$ and $C_{2}$ to the local wall shear stress $\tau$ was constructed in the following manner. Equation (3) gives the capping value of the length scale $\ell_{C A P}$, which begins at the nondimensional position $\mathrm{y}^{+}=C_{1}$ in the boundary layer and continues outward into the free stream. If this maximum length scale at a given position is related to the local boundary layer thickness $\delta$ through a constant $B$ (typically $B=0.09$ ), then the capping length scale is given as

$$
\ell_{\text {CAP }} \cong B \cdot \delta
$$

Empirical correlations for $\delta$ and $\tau$ in Eqs. (7) and (8) are taken from Ref. 6 and are appropriate for a flat plate in the range of Reynolds numbers being considered:

$$
\begin{gathered}
\frac{\delta}{x}=0.381 R e_{x}^{-2} \\
C_{f}=\frac{\tau}{\frac{1}{2} \rho v^{2}}=0.0592 R e_{x}^{-.2}
\end{gathered}
$$

Thus, an expression for the local capping length scale may be constructed as a function of the local skin friction or wall shear stress instead of boundary layer thickness: 


$$
\ell_{C A P}=\left(3.31 \cdot 10^{-6}\right) B C_{j}^{-3.5} \mathrm{y}^{*}
$$

Similar expressions that define the capping length scale as a function of boundary layer thickness, such as those of the Cebeci-Smith model, have been shown to be inadequate for separated flow situations. The edge of the boundary layer is difficult to determine in separated cases because of the recirculating flow. Equation (9) is defined independently of boundary layer thickness to improve the capability of PARC in predicting separated flows. For separated flows, Eq. (9) still will predict very large maximum length scales as the wall shear stress becomes small. To avoid this problem, Potapczuk ${ }^{3}$ used a weighted average for the shear stress

$$
\tau_{i}=.1\left|\tau_{t-2}\right|+.2\left|\tau_{i-1}\right|+.4\left|\tau_{1}\right|+.2\left|\tau_{t+1}\right|+.1\left|\tau_{1+2}\right|
$$

The subscripts in Eq. (10) refer to the computational grid point locations along the wall bounding the turbulent flow.

Equations (3) and (9) may be used to give the desired relation among $C_{1}, C_{2}$, and shear stress (skin friction)

$$
\frac{C_{1}}{C_{2}}=\left(3.31 \cdot 10^{-6}\right)\left(\frac{B}{K}\right) C_{f}^{-3.5}
$$

Equation (11) shows that if $B$ and $K$ are constants, the ratio of $C_{1}$ to $C_{2}$ is a function of the shear stress. If either $C_{1}$ or $C_{2}$ remains a constant, the other will be simply determined by Eq. (11) and will still allow their ratio to be a function of a local flow parameter such as shear stress.

\section{Thomas Model for Unbounded Flows}

The standard algebraic turbulence model in PARC is based on the work of Thomas. ${ }^{7}$ Although the Thomas model in PARC can be applied to all flows, it was optimized for free shear layers, which are unbounded flows where, for example, a jet may be mixing with a slower flow. Its capability for calculating wall-bounded turbulent flows has been questioned. The unbounded part of the Thomas model calculates eddy viscosity in free shear layers by first determining a length scale

$$
\ell=\frac{\ell_{o}\left[\operatorname{Max}\left(\left|u_{j}\right|\right)-\operatorname{Min}\left(\left|u_{j}\right|\right)\right]}{\omega_{c}}
$$

where $u_{j}$ is the velocity in a section, $\omega_{c}$ is the maximum vorticity in the section, and $\ell_{0}$ is a constant. Prandtl's mixing length hypothesis is used to determine turbulent viscosity as was done for the MML- bounded flow model. The Thomas-bounded flow model (not used for the current model) is detailed in Ref. 7.

\section{Linking of Bounded and Unbounded Models}

As mentioned previously, many propulsion flow cases will have both turbulent wall-bounded flow regions and unbounded regions. The current turbulence model will employ the MML model for the former and the Thomas model for the latter. The two models must also be linked appropriately to provide a smooth transition from the bounded to unbounded regions in the flow field. Figure 1 depicts a flow situation having both a wall boundary layer and a free shear layer. In the current model, the MML model will calculate turbulent viscosity from the wall out to the nondimensional position $\mathrm{y}^{+}=C_{3}$, which is roughly the edge of the wall boundary layer and can be defined by the local MML capping length scale multiplied by a constant. The Thomas model will be used in the unbounded region from the nondimensional position $y^{+}=$ $C_{4}$ (also defined as a constant times the local MML capping length) out into the free stream. In the transition region between $C_{3}$ and $C_{4}$, a linear function is used to determine the turbulent viscosity

$$
\mu_{\mathrm{tr}}=\frac{\mu_{\mathrm{MML}}\left(C_{4}-y^{+}\right)+\mu_{\mathrm{Th}}\left(y^{+}-C_{3}\right)}{C_{4}-C_{3}}
$$

The resulting combined model will be referred to as the MMLT model for the rest of this discussion.

\section{Validation of the MMLT Model}

\section{Calculation of Flow Over a Flat Plate}

The PARC code with the MMLT model was applied to a flow over a flat plate $(\mathrm{M}=0.2)$ to determine appropriate values for $C_{1}, C_{2}, C_{3}$, and $C_{4}$. Since $C_{1}$ and $C_{2}$ are related by $\mathrm{Eq}$. (11), five values of $C_{2}$ were 
determined by selecting five different positions in the wall boundary layer $\left(C_{1} / \delta^{+}\right)$where the MML capping length scale is reached. The parameter $C_{1} / \delta^{+}$is the ratio of the distance from the wall where $\ell_{\mathrm{CAP}}$ is reached to the boundary layer thickness and $\delta^{+}$is the nondimensional boundary layer thickness. Equations ( 3 ) and (6) can be used to obtain a relation between $C_{2}$ and the theoretical $C_{1} / \delta^{+}$.

$$
C_{2}=\frac{K}{B}\left(\frac{C_{1}}{\delta^{+}}\right)
$$

Note that the boundary layer thickness is used here only to optimize $C_{2}$. As mentioned previously, the MMLT model does not use the boundary layer thickness to calculate turbulent length scales or turbulent viscosity. The ratio $C_{1} / \delta^{+}$was varied from 0.3 to 0.7 to determine both an optimal $C_{2}$ and the sensitivity of the model to this parameter. The beginning of the transition region, $C_{3}$, was set as the nominal edge of the boundary layer, $10 \ell+{ }^{+} \mathrm{CAP}$, and the end of the transition region, $C_{4}$, was set to $20 \ell^{+}$CAP.

Figures 2 and 3 show the effect that variation of $C_{1} / \delta^{+}$had on the flat plate boundary layer predictions. The comparison of MMLT shear stress predictions to experimental data of Weighardt and Tillman ${ }^{8}$ in Fig. 2 indicates that the MMLT model matches the data best for larger values of $C_{1} / \delta^{+}$. The MMLT boundary layer velocity profiles are compared to experimental data from Ref. 9 at a plate Reynolds number of 4000000 in Fig. 3a and at a plate Reynolds number of 10000000 in Fig. 3b. The MMLT solutions demonstrate no significant differences among the different values of $C_{1} / \delta^{+}$. Other combinations of $C_{3}$ and $C_{4}$ were examined and did not change the solutions provided $10 \ell^{+}{ }_{\mathrm{CAP}} \leq C_{3}<C_{4}$. This was expected because $C_{3}$ and $C_{4}$ are the boundaries of the Iransition region from the wall-bounded MML model to the unbounded part of the Thomas model, and this flat plate flow has no free shear layer (unbounded) region. The following section discusses optimization of $C_{3}$ and $C_{4}$ for flow over a backward-facing step, a benchmark case for separating and reattaching flow.

This same flat plate case was analyzed with the three turbulence models already available in PARC and the results were compared with the MMLT predictions using $C_{1} / \delta^{+}=0.7$ (corresponding to $C_{2}=3.18$ ), $C_{3}=10 \ell{ }^{+} \mathrm{CAP}$, and $C_{4}=20 \ell+{ }^{C A P}$. The first of the three models was the Thomas model, the previously mentioned standard algebraic turbulence model in PARC. The second was the BaldwinLomax model recently installed by Sirbaugh. ${ }^{10}$ The third and only two-equation model was Chien's low Reynolds number $k-\varepsilon$ model ${ }^{11}$ modified by Nichols. ${ }^{12}$ Figure 4 shows a comparison of the shear stress predicted by these models with experimental data and Fig. 5 shows the boundary layer velocity profile comparisons at plate Reynolds numbers of 4000000 and 10000000 respectively. Only the original Thomas model results disagree strongly with the data.

The convergence histories for these flat plate cases are given in Table 1. The algebraic turbulence model solutions all took less than half the CPU time for the $k-\varepsilon$ solution, although all of the cases used the same 111 by 81 grid.

\section{Calculation of Elow Over a Backward-Facing Step}

Flow over a backward-facing step has also been analyzed with the PARC code and the MMLT model in order to optimize $C_{3}$ and $C_{4}$ and to determine the model's capability to calculate a separating and reattaching shear flow. Several MMLT backward-facing step cases were calculated (for varying $C_{1} / \delta^{+}, C_{3}$ and $C_{4}$ ) and compared. These PARC calculations modeled the experiment conducted by Driver and Seegmiller ${ }^{13}$ for a flow over a backward-facing step with $\mathrm{M}=0.128$ upstream of the step. The experiment was conducted in a low-speed wind tunnel with an expansion ratio (tunnel height after step to tunnel height before step) of 1.125 . The experimental geometry is shown in Fig. 6. The velocity profile measured at a position 4 step-heights upstream of the step in this experiment was used as the inflow boundary condition for the PARC calculations.

The following four combinations of $C_{3}$ and $C_{4}$ were used with $C_{1} / \delta^{+}=0.7$ and $C_{1} / \delta^{+}=0.3$ (eight total combinations) for the MMLT cases:

(1) $C_{3}=10 \ell+{ }_{\mathrm{CAP}}, C_{4}=15 \ell+{ }_{\mathrm{CAP}}$

(2) $C_{3}=10 \ell+{ }^{\mathrm{CAP}}, C_{4}=20 \ell+\mathrm{CAP}$

(3) $C_{3}=10 \ell+{ }_{\mathrm{CAP}}, C_{4}=40 \ell+{ }_{\mathrm{CAP}}$

(4) $C_{3}=20 \ell^{+}{ }_{\mathrm{CAP}}, C_{4}=40 \ell+{ }^{+} \mathrm{CAP}$

Shear stress predictions for the $\mathrm{C}_{1} / \delta^{+}=0.7$ cases appear in Fig. 7(a) and for the $\mathrm{C}_{1} / \delta^{+}=0.3$ cases in Fig. 7(b). All skin friction results undershoot the experimental data in the separated region just downstream of the step, and all MMLT predictions of reattachment length (location where $C_{f}=0$ ) are larger than the experimental value. The PARC MMLT reattachment predictions are given in Table 2. Eaton and Johnston 14 report that negative skin friction coefficients as large as -0.0012 are common for backwardfacing step flows. This is still smaller than the MMLT predictions but larger than the experimental data of Driver and Seegmiller. The most accurate reattachment predictions are provided by the solution with $C_{3}=10 \ell^{+}{ }^{C A P}$ and either $C_{4}=15 \ell^{+}{ }_{\mathrm{CAP}}$ or $C_{4}=20 \ell^{+} \mathrm{CAP}$. The MMLT 
skin friction predictions in the reattached region downstream of the step match the experimental data well. There is much less variation among the various combinations of $C_{3}$ and $C_{4}$ for cases with $C_{1} / \delta^{+}=0.7$ than with $C_{1} / \delta^{+}=0.3$.

Velocity profiles at $\mathrm{X} / \mathrm{H}=3,6,12$, and 20 appear in Figs. 8 and 9 for cases with $C_{1} / \delta^{+}=0.7$ and $C_{1} / \delta^{+}=$ 0.3 , respectively. The MMLT profiles overestimate the magnitude of the reversed velocity in the separated region $(\mathrm{X} / \mathrm{H}=3$ and $\mathrm{X} / \mathrm{H}=6)$ with the largest discrepancy for $C_{4}$ $=40 \ell+C$ AP. This corresponds to the skin friction results. The experimental velocities at $\mathrm{X} / \mathrm{H}=6$ indicate that the flow has already reattached at this position, although the experimental skin friction results indicate the flow is still separated, as Driver and Seegmiller report.

As was done for the flat plate case, backwardfacing step calculations were also obtained with PARC's three other turbulence models for comparison with the MMLT model. The MMLT solution with $C_{1} / \delta^{+}=0.7, C_{3}$ $=10 \ell+{ }^{+} \mathrm{CAP}$, and $C_{4}=20 \ell{ }^{+}$CAP was used for comparison with the Thomas, Baldwin-Lomax, and $k-\varepsilon$ solutions. Figure 10 shows a comparison of the skin friction predictions for these models and Table 3 gives the reattachment length predictions. Compared with the experimental data, the Thomas solution shows a much smaller negative skin friction before the reattachment and a much smaller positive skin friction after. The location of the reattachment is also severely overestimated.

The Baldwin-Lomax solution demonstrates the largest undershoot of the skin friction before the reattachment and largest overshoot after the reattachment of any of the models. The Baldwin-Lomax model behaves poorly for separated flows because the key function in the model, which describes the product of vorticity and length scale, is not easily determined for such situations. The Baldwin-Lomax model also predicts a smaller realtachment length than the experimental data.

It was anticipated that the $k-\varepsilon$ solution would provide the best match to the experimental data. The $k-\varepsilon$ solution does predict nearly the same reattachment length as the experimental data, but significantly overestimates the magnitude of the skin friction before and after the reattachment. The $k-\varepsilon$ skin friction prediction is the only one to reach a peak after the reattachment and then become smaller farther downstream. Avva, Smith, and Singhal 15 report the same skin friction behavior when applying a low Reynolds number k-E model to the same backward-facing step of Driver and Seegmiller. They found that the k$\varepsilon$ solution is very sensitive to the grid packing in the inner layer $\left(y^{+}<30\right)$ by varying the number of grid points in this region from 5 to 30 . The current study used the same grid for all the PARC backward-facing step cases with approximately 18 grid points in the inner layer.

As mentioned previously, the MMLT model undershoots the skin friction before the reattachment and predicts a larger reattachment length than was experimentally determined. However, the MMLT solution is the only one to closely match the experimental skin friction data downstream of the reattachment position.

Figure 11 presents a comparison of the velocity profiles at $\mathrm{X} / \mathrm{H}=3,6,12$, and 20 . The Thomas solution overall shows the poorest agreement with the experimental data as was the case with the flat plate examination. The other three models match the experimental data more accurately, but there is a large variation in their velocity profiles near the wall $(\mathrm{Y} / \mathrm{H}<1)$. Away from the wall, the Baldwin-Lomax solution predicts a larger free-stream velocity than do the other models or the experimental data.

The convergence histories for the backward-facing step cases are given in Table 4. These cases took much longer to converge than the flat plate cases. This was most likely due to the following: (1) the increased complexity of the separating and reattaching flow that caused the maximum allowable time step in PARC to be 30 times smaller than for the flat plate and (2) the PARC code's convergence rate becoming very slow for flows with a freestream Mach number near 0.1 or smaller $(0.128$ for the backward-facing step). The $k-\varepsilon$ solution took more than twice the iterations and more than seven times the CPU time to come to convergence than any of the algebraic model solutions.

\section{Conclusions}

The flat plate and backward-facing step flow computations with the PARC code have been valuable both in developing the MMLT model from its two existing algebraic turbulence model components and in assessing its capabilities and those of the other turbulence models in PARC.

All the turbulence models, except PARC's standard algebraic turbulence model, the Thomas model, provided accurate skin friction and boundary layer velocity profile predictions for the flat plate flow. None of the models agreed very well with the experimental skin friction and velocity profile data for the backward-facing step case in the separated region. Downstream of the reattachment, all the turbulence models, except for Thomas, show fair agreement with the experimental velocity profiles. The MMLT skin friction results match the experimental dita downstream of the reattachment much better than the odher 
models, including the $k-\varepsilon$ model which took seven times more CPU time than any of the algebraic models to converge.

Other flow cases will be investigated to determine the PARC code's capability to provide accurate propulsion flow predictions with the MMLT model. A single flow plug nozzle is being constructed and will be tested by NASA Langley Research Center to provide extensive data for code validation. This nozzle has been investigated with PARC using the Thomas and $k-\varepsilon$ models and will be investigated with PARC using the MMLT model.

\section{References}

1. Cooper, G.K. and Sirbaugh, J.R., "The PARC Distinction: A Practical Flow Simulator," AIAA Paper 90-2002, July 1990.

2. Beam, R.M., and Warming, R.F., "An Implicit FiniteDifference Algorithm for Hyperbolic Systems in Conservation-Law Form-Application to Eulerian Gasdynamic Equations," Journal of Computational Physics, Vol. 22, No. 1, 1976, pp. 87-110.

3. Potapczuk, M.G., "Navier-Stokes Analysis of Airfoils with Leading Edge Ice Accretions," Ph.D. Dissertation, Akron University, May 1989.

4. Pulliam, T.H., "Euler and Thin Layer Navier-Stokes Codes: ARC2D, ARC3D." Notes for Computational Fluid Dynamics User's Workshop, The University of Tennessee Space Institute, Tullahoma, Tennessee, (UTSI Publication E02-4005-023-84), 1984, pp. 15.115.85 .

5. Baldwin, B. and Lomax, H., "Thin Layer Approximation and Algebraic Model for Separated Turbulent Flows," AIAA Paper 78-257, January 1978.

6. Chapman, A. J., Fundamentals of Heat Transfer, Macmillan, New York, 1987.

7. Thomas, P.D., "Numerical Method for Predicting Flow Characteristics and Performance of Nonaxisymmetric Nozzles, Theory," NASA CR-3147, September 1979.

8. Weighardt, K. and Tillman, W., "On the Turbulent Friction Layer for Rising Pressure," NACA TM- 1314, 1951.
9. Clauser, F.H., "Turbulent Boundary Layers in Adverse Pressure Gradients," Journal of the Aeronautical Sciences, February 1954, pp. 91-108.

10. Sirbaugh, J.R. and Reichert, B.A., "Computation of a Circular-to-Rectangular Transition Duct Flow Field," AIAA Paper 91-1741, June 1991.

11. Chien, K.-Y., "Predictions of Channel and BoundaryLayer Flows with a Low-Reynolds-Number Turbulence Model," AIAA Journal, January 1982, Vol. 20, No. 1, pp. 33-38.

12. Nichols, R.H., "A Two-Equation Model for Compressible Flows," AIAA Paper 90-0494, January 1990.

13. Driver, D.M. and Seegmiller, H.L., "Features of a Reattaching Turbulent Shear Layer in Divergent Channel Flow," AIAA Journal, Vol. 23, No. 2, 1985, pp.163-171.

14. Eaton, J.K. and Johnston, J.P., "A Review of Research on Subsonic Turbulent-Flow Reattachment,"AIAA Journal, Vol. 19, No. 9, September 1981, pp. 1093-1100.

15. Avva, R.K., Smith, C.E., and Singhal, A.K., "Comparative Study of High and Low Reynolds Number Versions of $\mathbf{k}-\varepsilon$ Models," AIAA Paper 900246, January 1990. 
Table 1. Convergence histories for flat plate solutions.

\begin{tabular}{|c|c|c|}
\hline MODEL & ITERATIONS & $\begin{array}{c}\text { CRAY Y/MP } \\
\text { CPU TIME (s) }\end{array}$ \\
\hline MMLT & 4000 & 500 \\
Thomas & 4000 & 450 \\
Baldwin-Lomax & 4000 & 500 \\
Chien k- $\varepsilon$ & 5000 & 1100 \\
\hline
\end{tabular}

Table 2. Comparison of reattachment positions of the MMLT solutions.

\begin{tabular}{|c|c|}
\hline CASE & $\begin{array}{l}\text { REATTACHMENT } \\
\text { POSITION } \\
\text { (STEP HEIGHTS }, \mathrm{H})\end{array}$ \\
\hline Driver-Seegmiller Data & 6.250 \\
\hline $\begin{array}{c}\left(\mathrm{C}_{1} / \delta+=7, \mathrm{C}_{3}=10 \ell^{+} \mathrm{CAP}\right. \\
\left.\mathrm{C}_{4}=15 \ell^{+} \mathrm{CAP}\right)\end{array}$ & 7.353 \\
\hline $\begin{array}{c}\left(\mathrm{C}_{1} / \delta+=.7, \mathrm{C}_{3}=10 \ell+\mathrm{CAP}\right. \\
\left.\mathrm{C}_{4}=20 \ell+\mathrm{CAP}\right)\end{array}$ & 7.416 \\
\hline $\begin{array}{c}\left(\mathrm{C}_{1} / \delta+=.7, \mathrm{C}_{3}=10 \ell+\mathrm{CAP}\right. \\
\left.\mathrm{C}_{4}=40 \ell+\mathrm{CAP}\right)\end{array}$ & 7.566 \\
\hline $\begin{array}{c}\left(\mathrm{C}_{1} / \delta+=.7, \mathrm{C}_{3}=20 \ell+\mathrm{CAP}\right. \\
\left.\mathrm{C}_{4}=40 \ell+\mathrm{CAP}\right)\end{array}$ & 7.749 \\
\hline $\begin{array}{c}\left(\mathrm{C}_{1} / \delta+=7, \mathrm{C}_{3}=10 \ell+\mathrm{CAP}\right. \\
\left.\mathrm{C}_{4}=15 \ell+\mathrm{CAP}\right)\end{array}$ & 7.143 \\
\hline $\begin{array}{c}\left(\mathrm{C}_{1} / \delta+=.7, \mathrm{C}_{3}=10 \ell+\mathrm{CAP}\right. \\
\left.\mathrm{C}_{4}=20 \ell+\mathrm{CAP}\right)\end{array}$ & 7.429 \\
\hline $\begin{array}{c}\left(\mathrm{C}_{1} / 8+=.7, \mathrm{C}_{3}=10 \ell+\mathrm{CAP}\right. \\
\left.\mathrm{C}_{4}=40 \ell+{ }^{+} \mathrm{CAP}\right)\end{array}$ & 7.746 \\
\hline $\begin{array}{c}\left(\mathrm{C}_{1} / \delta+=.7, \mathrm{C}_{3}=20 \ell+\mathrm{CAP}\right. \\
\left.\mathrm{C}_{4}=40 \ell+\mathrm{CAP}\right)\end{array}$ & 8.786 \\
\hline
\end{tabular}

Table 3. Comparison of reattachment positions of PARC's other turbulence model solutions.

\begin{tabular}{|c|c|}
\hline CASE & $\begin{array}{c}\text { REATTACHMENT } \\
\text { POSITION } \\
\text { (STEP HEIGHTS,H) }\end{array}$ \\
\hline $\begin{array}{c}\text { Driver-Seegmiller Data } \\
\text { MMLT }\left(\mathrm{C}_{1} / \delta+=.7,\right. \\
\left.\mathrm{C}_{3}=10{ }^{+}{ }^{+} \mathrm{CAP}_{\mathrm{C}} \mathrm{C}_{4}=20{ }^{+}{ }^{+} \mathrm{CAP}\right)\end{array}$ \\
Thomas & 6.250 \\
Baldwin-Lomax & 7.416 \\
Chien k- $\varepsilon$ & 12.281 \\
\end{tabular}

Table 4. Convergence histories for backward-facing step solutions.

\begin{tabular}{|c|c|c|}
\hline MODEL & ITERATIONS & $\begin{array}{c}\text { CRAY Y/MP } \\
\text { CPU TIME (s) }\end{array}$ \\
\hline MMLT & 45,000 & 7100 \\
Thomas & 40,000 & 6000 \\
Baldwin-Lomax & 45,000 & 7000 \\
Chien k- $\varepsilon$ & 110,000 & 50,000 \\
\hline
\end{tabular}




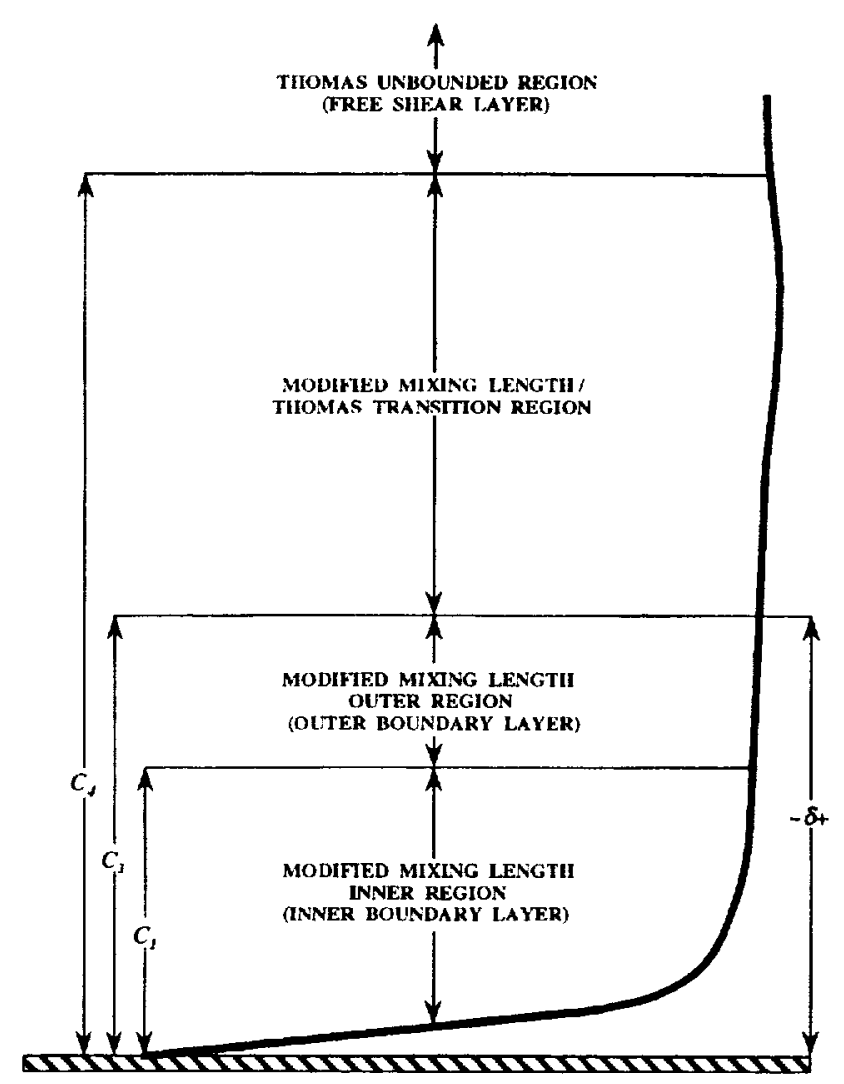

Figure 1.-Wall boundary layer and free shear layer.

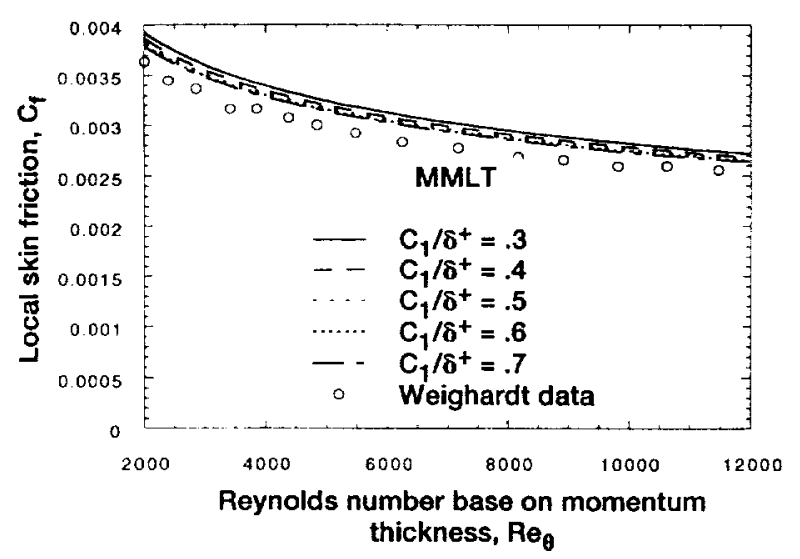

Figure 2.-MMLT flat plate skin friction predictions for varying $\mathrm{C}_{1} / \delta^{+}$.

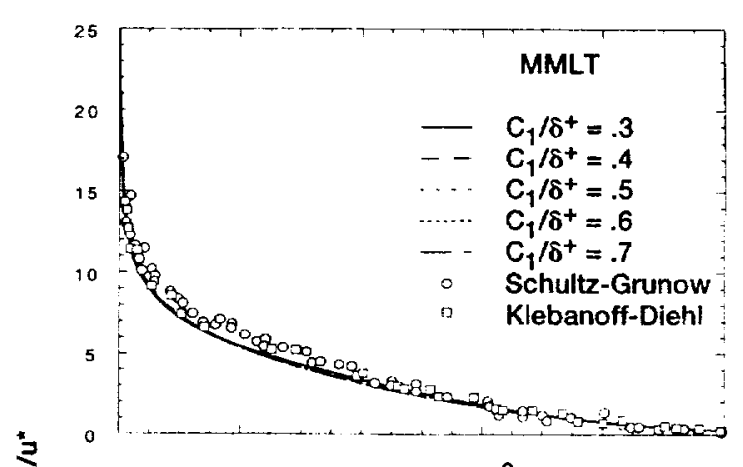

(a) $R e_{x}=4 \times 10^{6}$.

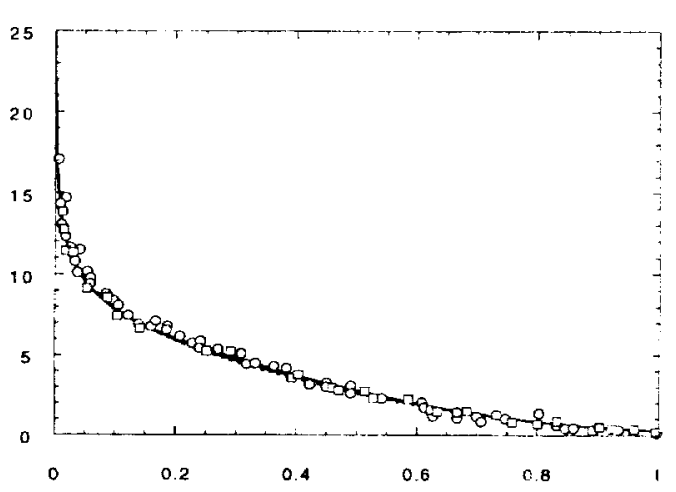

Position in boundary layer, $y / \delta$

(b) $R e_{x}=10^{?}$.

Figure 3.-MMLT flat plate boundary layer velocity profiles for varying $\mathrm{C}_{1} / \delta^{+}$.

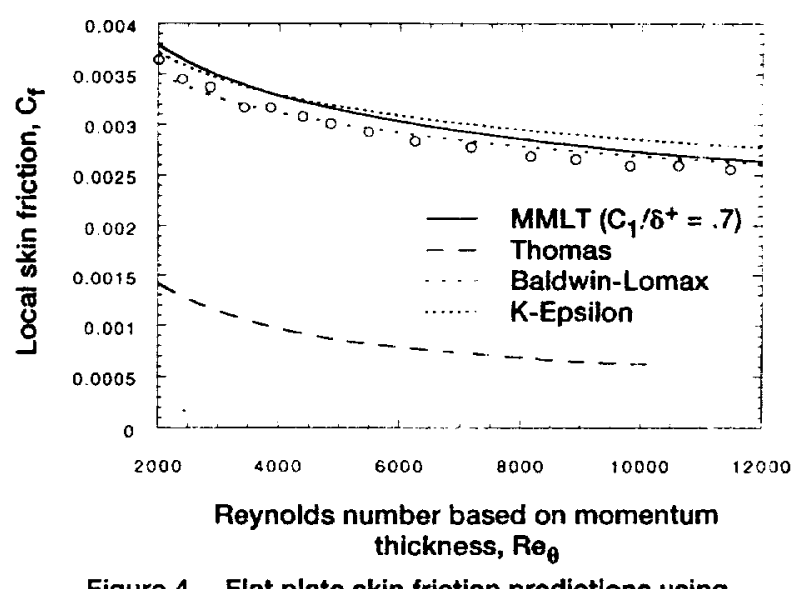

Figure 4.-Flat plate skin friction predictions using the different turbulence models in PARC. 


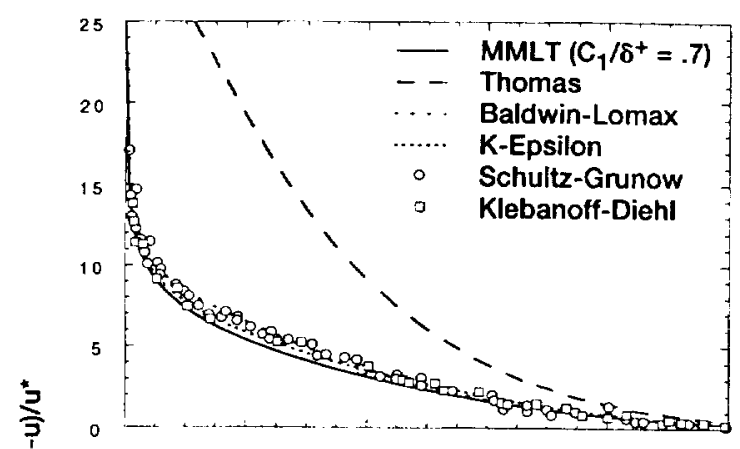

(a) $R e_{x}=4 \times 10^{6}$.

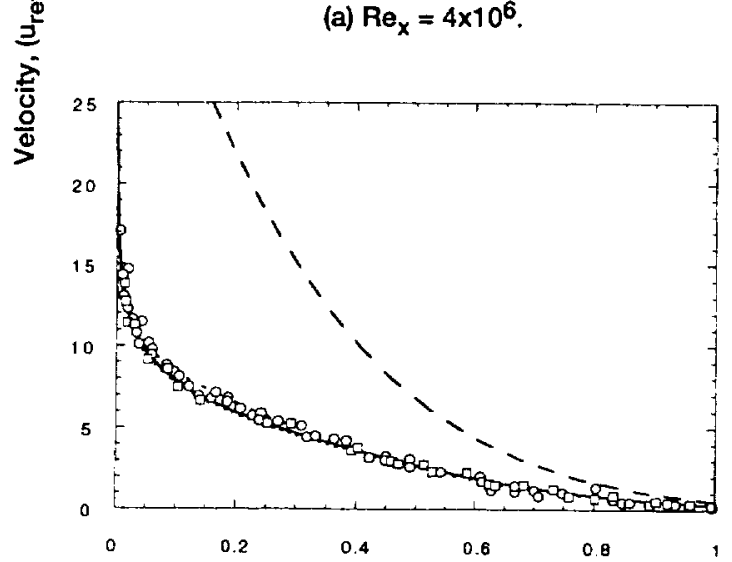

Position in boundary layer, $y / \delta$

(b) $\operatorname{Re}_{\mathrm{x}}=10^{7}$.

Figure 5.-Flat plate boundary layer velocity profiles using the different turbulence models in PARC.

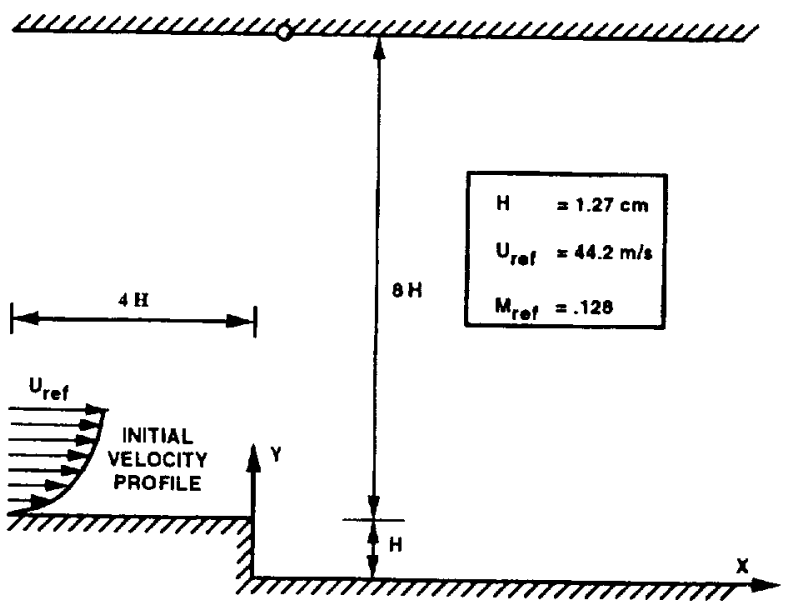

Figure 6.-Geometry of backward-facing step in experiment of Driver and Seegmiller.

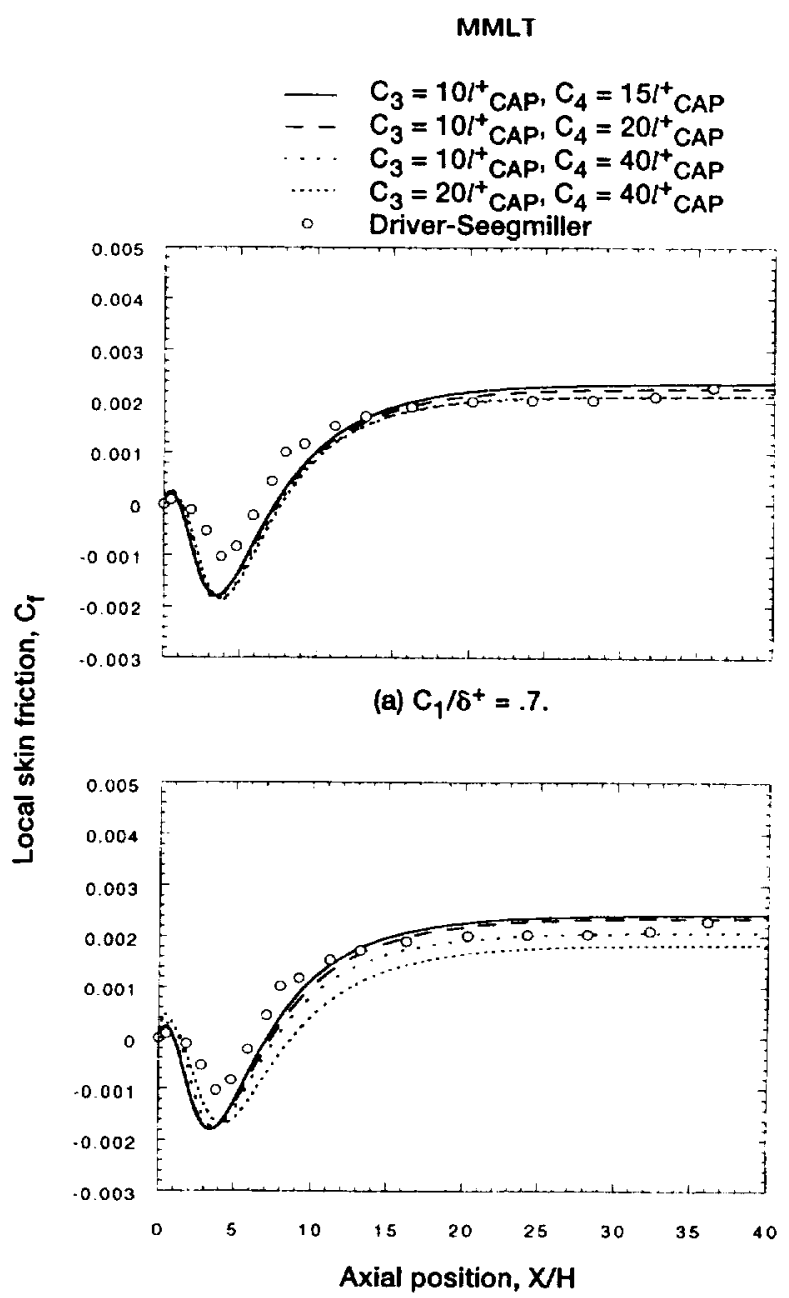

(b) $\mathrm{C}_{1} / 8^{+}=.3$.

Figure 7.-MMLT backward-facing step skin friction predictions with varying $\mathrm{C}_{3}$ and $\mathrm{C}_{4}$. 


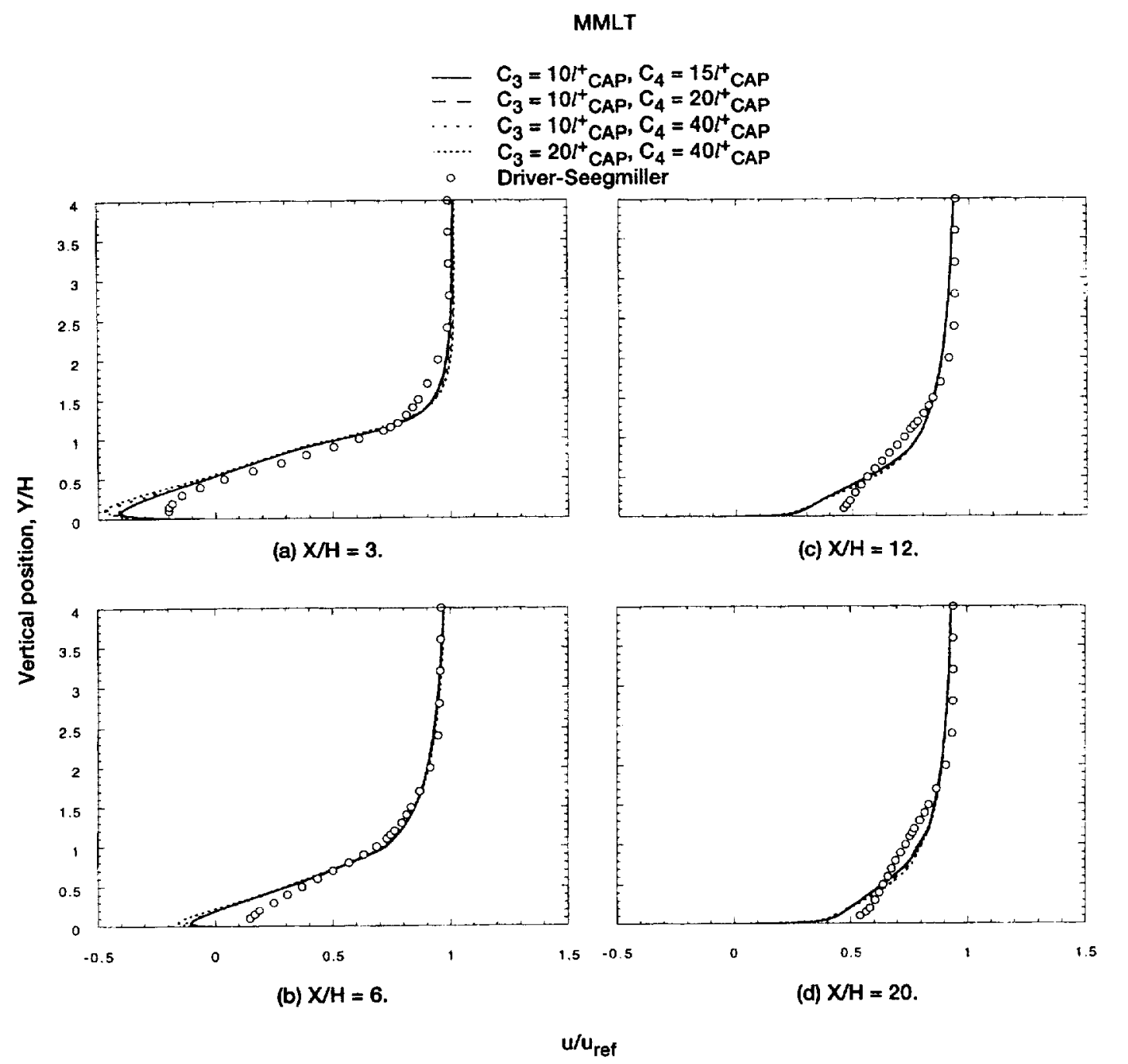

Figure 8.-MMLT backward-facing step velocity profile predictions for $C_{1} / \delta^{+}=.7$ and varying $C_{3}$ and $\mathrm{C}_{4}$. 
MMLI

CAP, $\mathrm{C}_{4}=15 l^{+} \mathrm{CAP}$

10+ ${ }^{+} \mathrm{CAP}, \mathrm{C}_{4}=20 \mathrm{I}^{+} \mathrm{CAP}$

$C_{3}=10 l^{+} \mathrm{CAP}, \mathrm{C}_{4}=40 l^{+} \mathrm{CAP}$

政

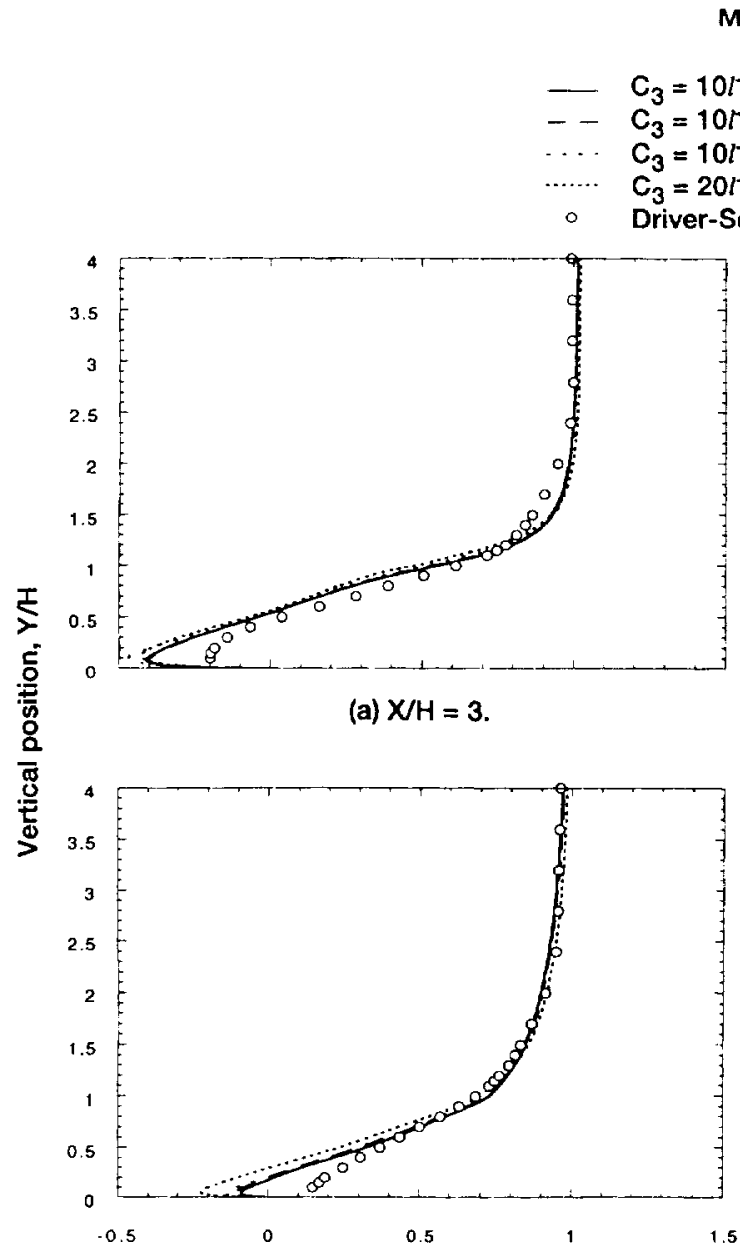

(b) $X / H=6$.

(a) $\mathrm{X} / \mathrm{H}=3$.

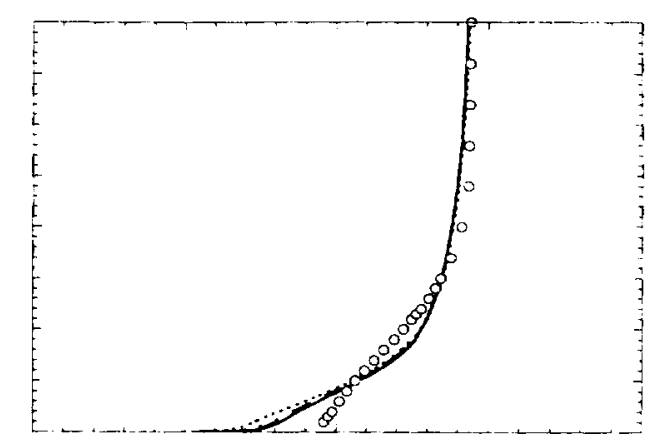

(c) $X / H=12$.

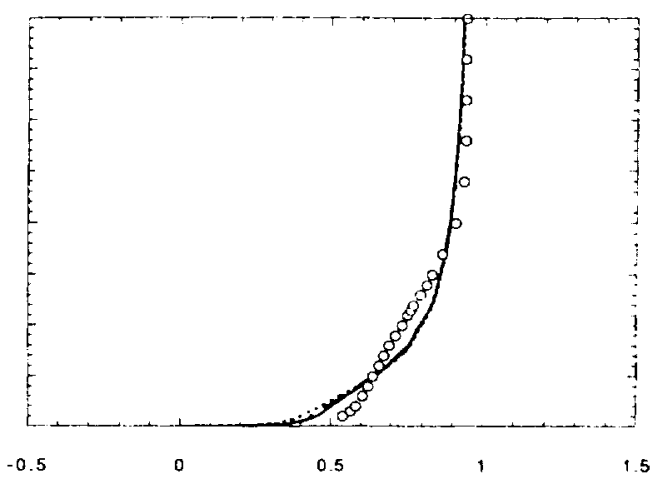

(d) $X / H=20$.

$u / u_{\text {ref }}$

Figure 9.-MMLT backward-facing step velocity profile predictions for $C_{1} / \delta^{+}=.3$ and varying $C_{3}$ and $\mathrm{C}_{4}$. 


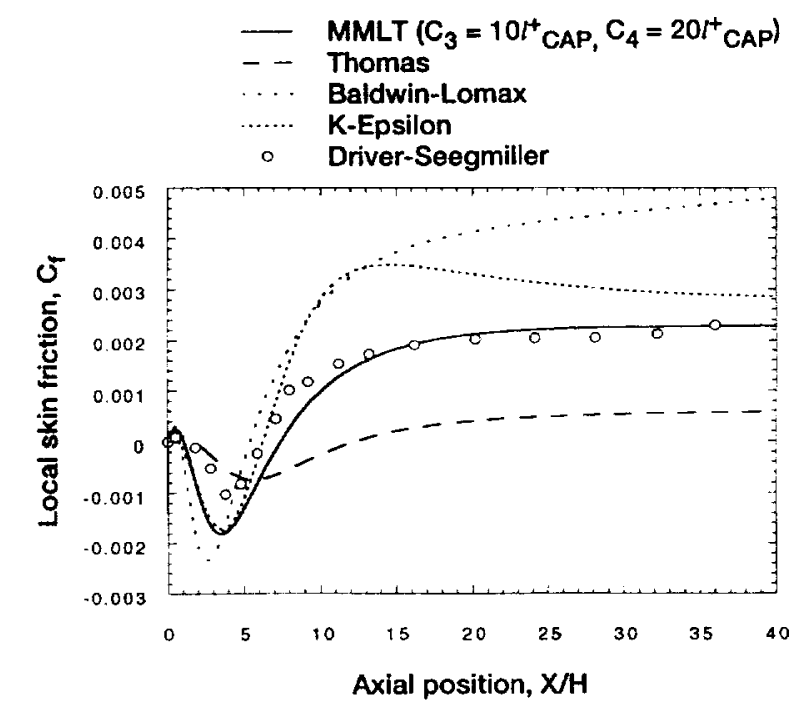

Figure 10.-Backward-facing step skin friction predictions using the different turbulence models in PARC.

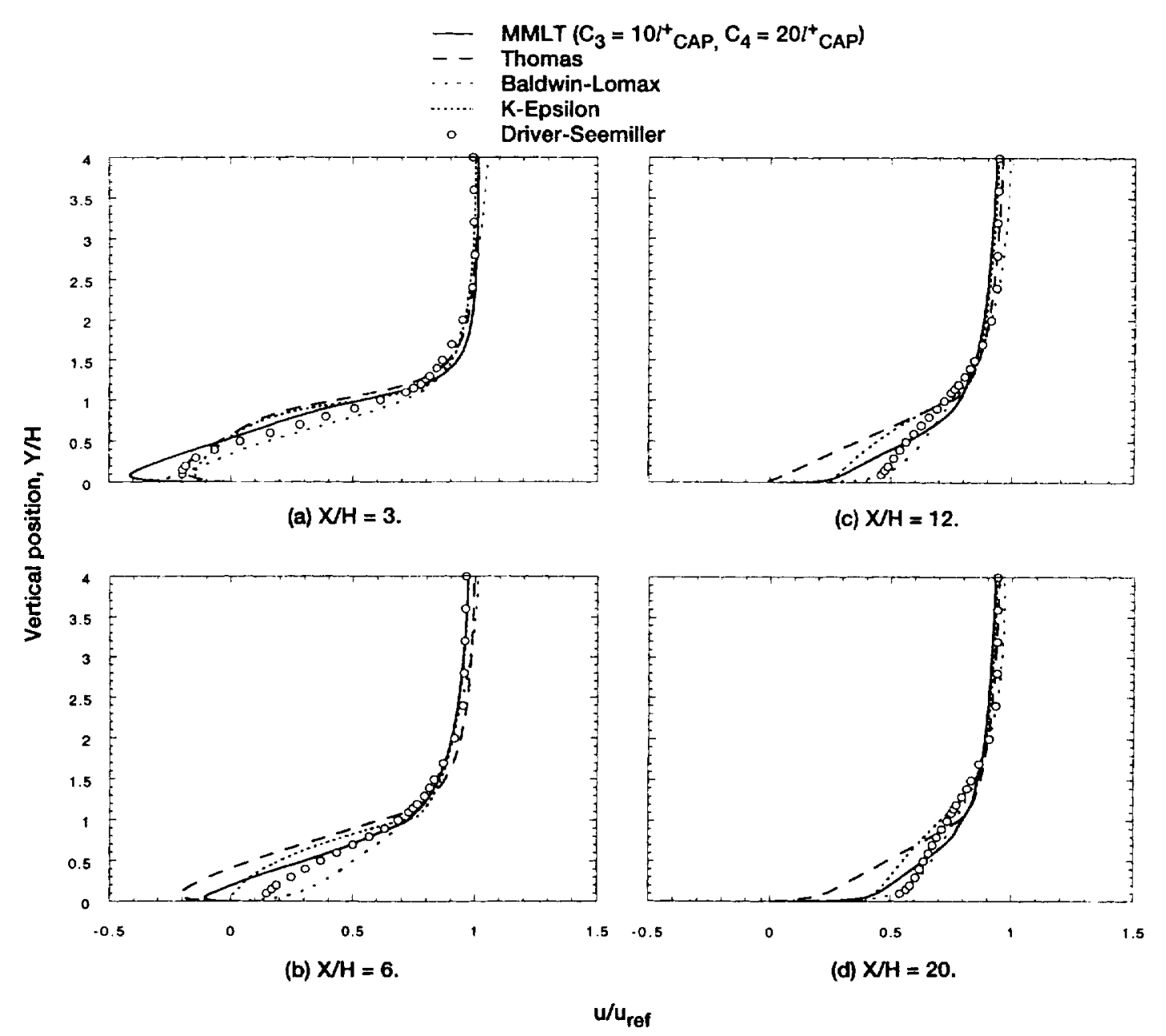

Figure 11.-Backward-facing step velocity profile predictions using the different turbulence models in PARC. 


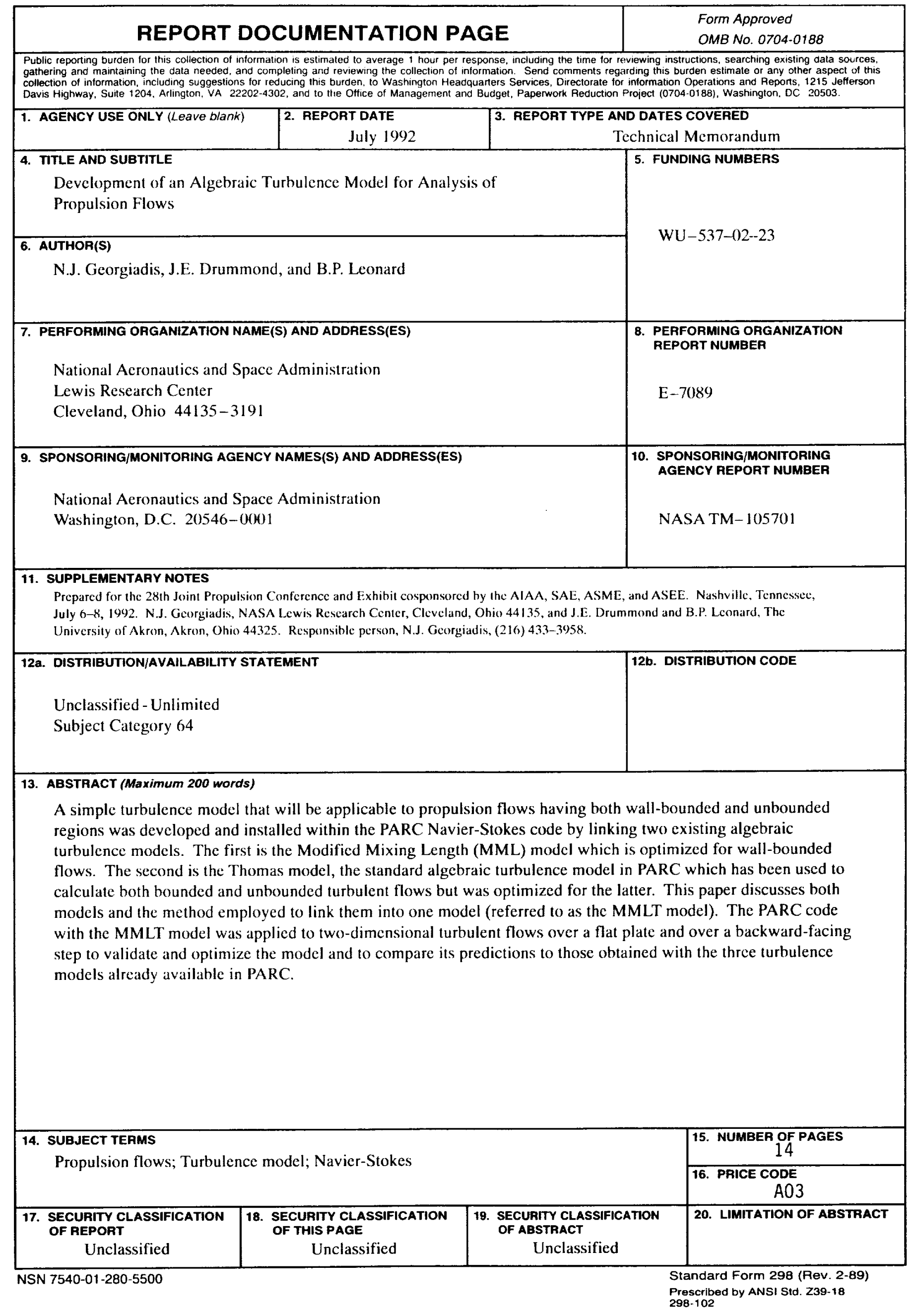

